\title{
Evaluation of transplacental transfer of mRNA vaccine products and functional antibodies during pregnancy and early infancy
}

Mary Prahl ( $\square$ mary.prahl@ucsf.edu )

University of California, San Francisco

Yarden Golan

University of California San Francisco

Arianna Cassidy

University of California San Francisco

Yusuke Matsui

Gladstone Institute

Lin Li

University of California San Francisco

\section{Bonny Alvarenga}

University of California, San Francisco

https://orcid.org/0000-0001-7922-9454

Hao Chen

University of California San Francisco

Unurzul Jigmeddagva

University of California San Francisco

Christine Lin

University of California San Francisco

Veronica Gonzalez

University of California San Francisco

Megan Chidboy

University of California San Francisco

Lakshmi Warrier

University of California San Francisco

\section{Sirirak Buarpung}

University of California San Francisco

Amy Murtha

UCSF

Valerie Flaherman

University of California San Francisco

Warner Greene 
J. David Gladstone Institutes

\section{Alan Wu}

University of California

Kara Lynch

UCSF

Jayant Rajan

University of California, San Francisco

\section{Stephanie Gaw}

University of California, San Francisco https://orcid.org/0000-0003-0891-6964

\section{Article}

Keywords: SARS-CoV-2, COVID-19, Pregnancy, Vaccine, Antibody, Neonatal Immunity, Neutralizing 47 Antibody, Phage Array, mRNA Vaccination, BNT-162b2, mRNA-1273, Placenta, Cord Blood

Posted Date: December 15th, 2021

DOI: https://doi.org/10.21203/rs.3.rs-1150427/v1

License: (1) (1) This work is licensed under a Creative Commons Attribution 4.0 International License. Read Full License

Version of Record: A version of this preprint was published at Nature Communications on July 30th, 2022. See the published version at https://doi.org/10.1038/s41467-022-32188-1. 
Evaluation of transplacental transfer of mRNA vaccine products and functional antibodies during

2 pregnancy and early infancy

4 Mary Prahl, M.D., Yarden Golan, Ph.D., Arianna G. Cassidy, M.D., Yusuke Matsui M.D., Ph.D.,

5 Lin Li, M.D., PhD, Bonny Alvarenga, Hao Chen, Ph.D., Unurzul Jigmeddagva, M.D., Christine Y.

6 Lin, Veronica J. Gonzalez, M.D, Megan A. Chidboy, Lakshmi Warrier, Sirirak Buarpung, D.V.M.,

7 Ph.D., Amy P. Murtha M.D., Valerie J. Flaherman, M.D., M.P.H., Warner C. Greene M.D.,

8 Ph.D., Alan H.B. Wu, Ph.D., Kara L. Lynch Ph.D., Jayant Rajan, M.D., Ph.D., Stephanie L. Gaw,

$9 \quad$ M.D., Ph.D

11 Author Affiliations

12 From the Department of Pediatrics, University of California, San Francisco (M.P., V.J.F.),

13 Division of Pediatric Infectious Diseases and Global Health, University of California, San

14 Francisco (M.P.), Department of Bioengineering and Therapeutic Sciences, University of

15 California, San Francisco (Y.G.), Department of Medicine, University of California, San

16 Francisco (L.W., B.A., J.R.), Weill Institute for Neurosciences, Division of Neurology, University

17 of California, San Francisco, CA (B.A.), Gladstone Center for HIV Cure Research, Gladstone

18 Institute, San Francisco, CA (Y.M., W.C.G.) Departments of Medicine and Microbiology and

19 Immunology, University of California, San Francisco (W.C.G.), Department of Laboratory

20 Medicine, University of California, San Francisco (A.H.B.W., K.L.L.), Center for Reproductive

21 Sciences, Department of Obstetrics, Gynecology, and Reproductive Sciences, University of

22 California San Francisco (A.G.C., L.L., H.C., U.J., C.Y.L., V.J.G., M.A.C., S.B., A.P.M., S.L.G.).

24 Co-Corresponding authors: 1) Mary Prahl- Division of Pediatric Infectious Diseases and Global 25 Health, Department of Pediatrics, University of California San Francisco, $55016^{\text {th }}$ St. $4^{\text {th }}$ Floor. 26 San Francisco, CA 94158. Phone (415) 514-0510, Email: mary.prahl@ucsf.edu 2) Stephanie L. 
27 Gaw-Division of Maternal-Fetal Medicine, Department of Obstetrics, Gynecology, and

28 Reproductive Sciences, University of California San Francisco, 513 Parnassus Ave, HSE16,

29 Box 0556, San Francisco, CA 94143. Phone: (415) 476-0535. Email: Stephanie.Gaw@ucsf.edu

31 Abstract

32 Studies are needed to evaluate the safety and effectiveness of mRNA SARS-CoV-2 vaccination

33 during pregnancy, and the levels of protection provided to their newborns through placental

34 transfer of antibodies. We evaluated the transplacental transfer of mRNA vaccine products and

35 functional anti-SARS-CoV-2 antibodies during pregnancy and early infancy in a cohort of 20

36 individuals vaccinated during pregnancy. We found no evidence of mRNA vaccine products in

37 maternal blood, placenta tissue, or cord blood at delivery. However, we found time-dependent

38 efficient transfer of IgG and neutralizing antibodies to the neonate that persisted during early

39 infancy. Additionally, using phage immunoprecipitation sequencing, we found a vaccine-specific

40 signature of SARS-CoV-2 Spike protein epitope binding that is transplacentally transferred

41 during pregnancy. In conclusion, products of mRNA vaccines are not transferred to the fetus

42 during pregnancy, however timing of vaccination during pregnancy is critical to ensure

43 transplacental transfer of protective antibodies during early infancy.

44

45 Keywords

46 SARS-CoV-2, COVID-19, Pregnancy, Vaccine, Antibody, Neonatal Immunity, Neutralizing

47 Antibody, Phage Array, mRNA Vaccination, BNT-162b2, mRNA-1273, Placenta, Cord Blood

48

49 Introduction

50 Growing evidence has shown that pregnant individuals are at higher risk for SARS-CoV-

51 2-related morbidity and mortality ${ }^{1-4}$. Despite this, vaccination uptake by pregnant individuals has

52 been slower than the general population ${ }^{5}$, in part because of maternal concern of adverse 
53 effects on the embryo or fetus, even with strong consensus recommendations for COVID-19

54 vaccination prior to or during pregnancy from several medical societies ${ }^{6}$. Pregnant individuals

55 were excluded from initial vaccine trials, and complete data on safety, efficacy, optimal timing of

56 the vaccine in pregnancy, or its impact on the fetus has been delayed ${ }^{7}$, which may impact

57 individual medical decision making. Current COVID-19 vaccines fully approved and under

58 emergency use in the United States include the mRNA vaccines BNT-162b2 and mRNA-1273,

59 which target the SARS-CoV-2 Spike protein and stimulate protective immune responses ${ }^{8,9}$. In

60 addition to protecting the mother against severe disease, vaccination during pregnancy may

61 protect the newborn through passive transfer of maternal immunoglobulin. SARS-CoV-2

62 infection and vaccination during pregnancy produces an $\lg G$ response that is transferred to the

63 fetus ${ }^{10-16}$. Evidence of newborn protection might help address maternal concerns about adverse

64 effects. However, detailed studies of the transplacental transfer of vaccine products and

65 vaccine-related antibody dynamics, functional properties, and persistence during infancy of

66 transferred SARS-CoV-2 antibodies are needed to provide such evidence.

67 We examined the transplacental transfer of mRNA vaccine products and humoral

68 responses using samples from pregnant individuals and their infants vaccinated with either

69 BNT-162b2 or mRNA-1273 mRNA vaccine during pregnancy.

71 Results:

72 Cohort: We evaluated 20 pregnant individuals who received COVID-19 mRNA vaccines during

73 pregnancy and their infants. Participants were vaccinated between December 2020 and April

74 2021. Gestational age at first dose ranged from 13 weeks to 40 weeks (mean 31.2, SD 5.9

75 weeks). Nineteen participants delivered live, singleton infants between January 2021 through

76 April 2021 at gestational ages ranging from 37.4 to 41.1 weeks (mean 39.2, SD 1.1 weeks).

77 One participant who was vaccinated at 13 weeks had a termination of pregnancy due to a lethal

78 skeletal dysplasia of genetic etiology at 20.4 weeks. Eight participants received BNT-162b2 
79 (Pfizer-BioNTech) and twelve received mRNA-1273 (Moderna) vaccines. Eighteen participants

80 received both vaccine doses prior to delivery, and two participants received the second dose

81 after delivery. The time from first mRNA vaccine dose ranged from 6-97 (mean 51, SD 24.3)

82 days prior to delivery, time from the second dose ranged from 2-75 (mean 32, SD 21.3) days

83 prior to delivery, and in two participants 15 and 21 days after delivery. No participants received

84 a $3^{\text {rd }}$ dose prior to delivery. Infants born to vaccinated mothers were followed up at convenience

85 time points ranging from age 3 weeks to 15 weeks of life (mean 8.3, SD 3.2). Further

86 demographic data is detailed in Table S1.

87

88 Vaccine mRNA products do not cross the placenta

To determine the transplacental transfer of mRNA vaccine derived products, we

90 examined available maternal blood at delivery, placenta tissue, and cord blood for Spike protein

91 by Western blot and vaccine mRNA by PCR. All available delivery samples (maternal blood,

92 placental tissue, and cord blood) were negative for Spike protein by Western blot (Supp Figure

93 1, Supp Table 3) and did not have detectable levels of vaccine mRNA by PCR (Suppl Table 3).

94 Together, this indicates that products of mRNA vaccination do not reach the fetus after

95 vaccination during pregnancy at readily detectable levels.

\section{7 mRNA vaccination in pregnancy leads to a robust antibody response}

Similar to prior studies ${ }^{14,15,17}$, we found that mRNA vaccination during pregnancy led to

99 an increase in anti-SARS-Cov-2 lgG following dose $1(n=7$, mean 388.6, SD 224.8 RFU) and an

100 even further robust increase after vaccination dose 2 ( $n=12$, mean 3214, SD 1383 RFU). Anti-

101 SARS-CoV-2 IgM (n=7, mean 53.3, SD 50.2 RFU) was detected in two maternal participants

102 following dose 1, but only 1 participant following dose 2 ( $n=12$, mean 23.8, SD 17 RFU, Fig 1). 
We then evaluated the transplacental transfer of maternally derived anti-SARS-CoV-2

107 IgG antibodies to their infants. Maternal blood at delivery was available in 19/20 participants and 108 cord blood was available in 17/20 participants. Anti-SARS-CoV-2 lgG was detectable in $94.7 \%$ 109 (18/19) of maternal blood samples at delivery (mean 3235, range [10, 7811] RFU). Anti-SARS110 CoV-2 IgG was detectable in 88.2\% (15/17) cord blood samples (mean 2243, range [2, 4959] 111 RFU). One participant received one mRNA vaccine dose 9 days prior to delivery, and both the 112 maternal and cord blood were negative for IgG at the time of delivery. Another participant 113 received two doses of mRNA vaccine (23 and 2 days) prior to delivery and maternal blood was 114 positive at 55 RFU (positive cutoff $>50$ RFU), however cord blood lgG was negative (Figure 2A).

115 Maternal and cord blood anti-SARS-CoV-2 lgG levels were moderately correlated, but not 116 statistically significant $\left(p=0.074, R_{s}=0.446\right.$, Fig $\left.2 A\right)$. All cord blood samples were anti-SARS117 CoV-2 IgM negative. We next evaluated the transplacental transfer of neutralizing antibody titers by a label119 free surrogate neutralization assay (sVNT) from mother to cord blood. Maternal and cord blood 120 at delivery had robust neutralizing responses (maternal $n=17$, mean 220.2, range [0, 422]. Cord 121 blood $n=16$, mean 296.6, range [0, 485], Fig 2B). All mother-infant dyads with positive IgG 122 serology at delivery had detectable transplacental transfer of neutralizing antibodies with the 123 exception of one pair in which the mother was borderline IgG positive at delivery and cord blood 124 was negative, for which both maternal and cord blood were negative for neutralizing titers (Fig 125 2B). However, maternal and cord blood neutralizing titers were not significantly correlated $126\left(p=0.361, R_{s}=-0.244\right.$, Fig 2B). Taken together, this indicates that maternal mRNA vaccination 127 induces functional neutralizing antibodies that are transferred to the infant. 
A subset of infants was sampled at convenience timepoints during follow up (infant $\mathrm{n}=11$, weeks of life range $[3,15]$ mean 8.3 weeks). Anti-SARS-CoV-2 IgG levels were positive in $81.8 \%$ of infants at follow-up (9/11 infants, mean 1290, range [1, 3225] RFU, Fig 2A), with one

134 infant still IgG positive at 12 weeks of age (Fig 2C). The two infants that were IgG negative at

135 follow up were both born to mothers who received only one vaccine dose prior to delivery (6 and 1369 days, respectively). One of these infants did not have paired maternal or cord blood available 137 at the time of delivery for comparison, and the other was IgG negative in cord blood. Maternal 138 and infant follow-up anti-SARS-CoV-2 lgG levels were not significantly correlated; however, 139 cord blood and infant follow-up IgG levels were significantly associated $\left(p=0.492, R_{s}=0.249\right.$ and $140 \mathrm{p}=0.021, \mathrm{R}_{\mathrm{s}}=0.76$, respectively, Fig 2A). All infants were IgM negative at the time of follow up. All infants with available IgG positive samples at follow up had detectable neutralizing 142 titers ( $n=8$, mean 154, range [41-256], Fig 2B). Maternal and infant follow-up neutralizing titers were not significantly correlated, as well as cord and infant follow up neutralizing titers $(p=0.665$,

$144 \quad R_{s}=-0.191$ and $p=0.662, R_{s}=0.214$, respectively, Fig 2B).

To compare the rate of decay of IgG antibody levels in mothers and their infants, we evaluated 5 dyads with paired maternal and infant blood samples on the same day at the time of

147 follow-up (range 3-9 weeks post-delivery). Maternal antibody IgG levels decreased faster in 148 mothers than infants (mean delta -974 RFU and -670 RFU, respectively. Fig 2E) at the follow up

149 timepoint. Taken together this indicates, maternally-derived functional vaccine induced 150 antibodies persist at high levels in newborns through early infancy during a critical time of

151 immune vulnerability and may decay slower than maternal lgG antibodies. 
We assessed the relationship of anti-SARS-CoV-2 IgG levels to neutralizing antibody

levels. We found a strong correlation between $\lg G$ and neutralizing titers in maternal plasma at delivery $\left(R_{s}=0.744, p=0.0012\right)$ and infant follow up $\left(R_{s}=0.738, p=0.046\right)$ timepoints, but no significant association between IgG and neutralizing titers in cord blood $\left(R_{s}=0.121, p=0.656\right.$, Figure 3).

We then evaluated the impact of timing of vaccination on maternal antibody levels at delivery. We found no statistically significant correlation between maternal IgG levels at delivery and time since dose $1\left(R_{s}=-0.335, p=0.160\right)$ and gestational age at delivery $\left(R_{s}=0.270, p=0.265\right.$, Fig 4A,B). This lack of correlation appeared to be driven by two participants that had low or 163 absent levels of antibodies at delivery and received their first dose of vaccine within 30 days of 164 delivery. We then evaluated neutralizing titers in those participants with known detectable $\lg G$ 165 levels at delivery and found that maternal neutralizing titers at delivery trended with days since 166 vaccine dose 1 but was not statistically significantly $\left(R_{s}=-0.422, p=0.093\right)$, and maternal neutralizing titers at delivery was not associated with gestational age at dose $1\left(R_{s}=0.074\right.$, $p=0.780$ ). One participant was borderline IgG positive at delivery (vaccinated within 30 days of delivery) and did not have detectable neutralizing titers at delivery (Fig 4C,D).

To assess facilitated antibody transfer, we evaluated cord-to-maternal antibody IgG and 171 neutralization titer ratios by time since vaccination and gestational age. We found that lgG ratios 172 were highly correlated with both time since first maternal vaccination dose and gestational age 173 at first dose $\left(R_{s}=0.917, p<0.0001\right.$ and $R_{s}=-0.739, p=0.002$, respectively. Fig 4E,F $)$. However, 174 neutralization titer cord-to-maternal ratios by time since first vaccination dose and gestational 175 age at first dose were not significantly associated $\left(R_{s}=0.366, p=0.179\right.$ and $R_{s}=-0.032, p=0.913$, 176 respectively, Figure $4 \mathrm{G}, \mathrm{H})$. Together, this may indicate that timing of vaccination in pregnancy is

177 critical for maternal-fetal antibody transfer, and functional neutralizing antibodies are

178 differentially transferred to the fetus as compared to total anti-SARS-CoV-2 IgG during 179 gestation. 
mRNA vaccination leads to a unique SARS-CoV2 Spike protein antibody epitope binding signature

We next investigated antibody linear epitope binding and transplacental transfer using the PhIP-seq/VirScan SARS-CoV-2 Spike protein phage display array in mother-infant dyads at the time of birth (Figure 5). We found that timing of vaccination was important for the transplacental transfer of Spike protein epitope binding antibodies. Two mother-infant dyads had minimal Spike protein specific epitope binding. The first dyad only received one dose of mRNA vaccine 9 days prior to delivery, and the other dyad received the second vaccine dose 2 days prior to delivery.

We found high levels of SARS-CoV-2 Spike protein epitope binding in 4 major peaks we 191 designate as regions 1-4 (Figure 5A). Region 1 overlays the carboxy terminal of the $\mathrm{N}$-terminal 192 domain. Region 2 overlaps with key residues for the S1/S2 cleavage site. Regions 3 and 4 are 193 within S2, flanking the fusion loop and the transmembrane portion of the Spike protein, 194 respectively. However, we found minimal binding in the receptor binding domain (RBD) of Spike 195 protein. Prior evaluation using the PhIP-seq/VirScan SARS-CoV-2 epitope phage array during SARS-CoV-2 infection demonstrated similar binding in regions 3 and 4, however in SARS-CoV-

1972 infection there was minimal binding in regions 1 and 2 demonstrating that antibody epitope 198 binding in these regions may be unique to vaccination ${ }^{18}$. Additionally, there is proportional 199 representation of linear epitope binding across the SARS-CoV-2 Spike protein proteome 200 between mothers and infants (Figure 5B). Taken together, SARS-CoV-2 antibody linear epitope 201 binding after vaccination during pregnancy shows similar patterns, with multiple 202 immunodominant regions found in the majority of mothers and infants. Some of these regions 203 are unique to vaccination and not observed during natural infection ${ }^{18-20}$.

\section{Discussion}


Among twenty women who received the COVID-19 mRNA vaccine during pregnancy,

207 our study found no evidence of transplacental transfer of mRNA vaccine products but did find

208 high levels of functional vaccine-derived antibodies that transferred to the infant at delivery and

209 persisted during early infancy. Additionally, we identified high levels of epitope binding in two

210 regions of Spike protein unique to SARS-CoV-2 vaccination ${ }^{18}$. These data may address some of

211 the many unanswered questions regarding COVID-19 vaccination in pregnancy: including the

212 dynamics of antibody production in the pregnant immune state, and the optimal timing of

213 immunization in pregnancy to impart passive immunity to the newborn during the vulnerable first

214 few weeks of infancy.

215 Uptake of COVID-19 vaccination in pregnancy has been slow ${ }^{5}$, and reasons for vaccine

216 hesitancy are likely multifactorial - but theoretical concerns that vaccine mRNA could cross the

217 placenta have been raised. We found no evidence of mRNA vaccine products in any of our

218 delivery samples. Additionally, no infants in our study had a fetal immune response to Spike

219 protein as demonstrated by a negative anti-SARS-CoV-2 IgM antibody in cord blood and infant

220 follow up samples. This further supports the lack of transfer of vaccine products, as only lgG is

221 transferred from the mother, and IgM production would indicate an endogenous fetal immune

222 response which has rarely been seen in natural infection with SARS-CoV-2 during pregnancy

223 16,21-23. This provides additional reassurance that mRNA vaccination is safe during pregnancy.

224 We found that the timing of immunization during pregnancy is important to ensure trans-

225 placental transfer of protective antibodies to the neonate, and during critical windows of immune

226 vulnerability during early infancy. Consistent with prior studies showing robust immune

227 responses to mRNA vaccination ${ }^{14,15,17}$, we found high levels of IgG after two doses of mRNA

228 vaccine. However, completion of the vaccination series well before delivery was important to

229 ensure transfer of antibodies to the infant. Two mothers only received one vaccine dose prior to

230 delivery and did not transfer antibodies as demonstrated by the lack of antibodies in cord (in one

231 with available cord blood) and in both infants at follow-up. Additionally, neutralizing antibodies 
were not transferred in a mother who received her second dose of vaccine 2 days prior to delivery. All evaluated mothers who received both doses during pregnancy and with the second dose greater than 9 days prior to delivery transferred IgG and neutralizing antibodies to their infants. Consistent with early studies of antibody transfer after COVID-19 vaccination in pregnancy, most of our participants were vaccinated in the third trimester of pregnancy. Larger studies of individuals vaccinated prior to pregnancy and in the first and second trimester are needed to understand persistence and waning of vaccine-induced immune responses.

Additionally, we believe we are the first to report that infants in the first few months of life continued to have maternal vaccine-derived anti-SARS-CoV-2 antibodies that were functional

241 as demonstrated by high levels of neutralizing antibodies presenting infants up to 12 weeks of 242 age. This is consistent with known persistence of maternally-derived antibodies from other 243 vaccinations including pertussis, rubella, varicella ${ }^{24-26}$. Additionally, we have previously found 244 persistence of anti-SARS-CoV-2 IgG antibodies in infants after natural infection up to 6 245 months $^{16}$. However, the functional capability of these antibodies as compared to anti-SARS246 CoV-2 vaccination-derived antibodies is unknown. Further evaluation of the longitudinal 247 persistence of maternal vaccine-derived antibodies during infancy will be critical to determine 248 optimal timing of COVID-19 vaccination in infancy.

249 Evaluation of paired maternal and baby samples at post-partum follow up timepoints 250 showed a faster decline in maternal IgG antibody levels than infants, suggesting that 251 persistence of maternally-derived antibody may be prolonged for infants. Differences in renal 252 excretion and neonatal Fc receptor (FcRn) expression, which is involved in antibody 253 degradation ${ }^{27}$ in the infant as compared to adults, could underly these differences and should be 254 explored further.

Consistent with observations in non-pregnant adults, we found that lgG levels in mothers 256 at delivery, and at infant follow-up were highly correlated with neutralizing titers ${ }^{28}$. However, 257 cord blood IgG levels did not correlate with neutralizing titers. Moreover, IgG cord-to-maternal 
ratios, which represent a proxy of maternal to fetal antibody transfer, were highly correlated with

259 timing of vaccination (gestational age and days since the first dose), but cord-to-maternal

260 neutralizing titer ratios were not significantly associated with time since vaccination nor

261 gestational age. During gestation there is facilitated transfer of maternally derived antibodies

262 through the binding of the neonatal Fc receptor in the synctiotrophoblast layer ${ }^{29}$. Differences in

263 glycosylation ${ }^{30,31}, \mathrm{FcR} / \mathrm{FcRn}$ binding affinity ${ }^{17,32}$, preferential lgG subclass transfer ${ }^{33,34}$ may be

264 different in functional neutralizing antibodies as compared to total IgG antibody transfer.

265 However, a limitation of this study is the majority of participants were vaccinated in the third

266 trimester. Further investigations on factors that influence the transport of functional antibodies

267 across trimesters are needed to understand antibody dynamics and optimal transfer of

268 protective antibodies to infants.

269 Using a PhIP-seq/VirScan SARS-CoV-2 Spike protein phage array we were able to

270 compare linear epitope antibody binding in mothers and their infants. Consistent with IgG and

271 neutralizing antibody evaluation, timing of vaccination was critical to ensure the transplacental

272 transfer of antibodies to the infant. Additionally, we identified unique regions of antibody epitope

273 binding in our vaccinated cohort that were not identified using the same phage library in a prior

274 evaluation of a cohort of SARS-CoV-2 infected individuals ${ }^{18}$. One of these regions included the

275 carboxy terminal of the N-terminal domain, with other work having shown that the $\mathrm{N}$-terminal

276 domain is targeted by neutralizing antibodies against Spike protein ${ }^{35}$. We did not see significant

277 binding in the receptor binding domain (RBD), which may be attributable to the fact that the

278 phage display library displayed short, linear peptides while antibodies targeting RBD are known

279 to target conformational epitopes. Lastly, we found that the same immunodominant regions

280 targeted by antibodies targeting the Spike protein in both mothers and infants.

281 In summary, this work provides further evidence that mRNA vaccination is safe in

282 pregnancy and demonstrates that it generates time-dependent protective, functional antibody

283 responses in mothers and infants that persist during early infancy. 


\section{Methods}

286 Cohort and Sample collection: The University of California San Francisco (UCSF) institutional 287 review board approved the study (20-32077). Written informed consent was obtained from all 288 participants. We enrolled 20 pregnant individuals who were vaccinated with either BNT-162b2 289 or mRNA-1273 mRNA vaccines. Pregnant individuals were followed through delivery, and their 290 infants were followed up to 12 weeks of life. Maternal blood was collected during pregnancy 291 (pre-vaccine, 3-4 weeks post-dose 1, 4-8 weeks post-dose 2). During delivery, maternal blood, 292 placenta tissue, and cord blood was collected. Infant follow-up blood was collected at 293 convenience timepoints. Whole blood was immediately added to RNAlater in a 1:1.3 ratio. 294 Plasma was isolated from whole blood by centrifugation and immediately cryopreserved. Full295 thickness placental biopsy was collected within 1 hour of delivery, washed three times with 296 phosphate buffered saline, and preserved in RNAlater.

SARS-CoV-2 plasma serology. Anti-SARS-CoV-2 plasma IgM and IgG antibodies were measured using the Pylon 3D automated immunoassay system (ET Healthcare, Palo Alto, CA). In brief, quartz glass probes are pre-coated with either affinity purified goat anti Human $\lg \mathrm{M}(\lg \mathrm{M}$ capture) or Protein G (IgG capture) are dipped into diluted patient sample. Samples are washed, and then the probe is dipped into the assay reagent containing both biotinylated recombinant spike protein receptor binding domain (RBD) and nucleocapsid protein (NP). After a washing, the probe is incubated with a Cy®5-streptavidin (Cy5-SA) polysaccharide conjugate reagent, allowing for cyclic amplification of the fluorescence signal. The background corrected signal is reported as relative fluorescent units (RFU) which is proportional to the amount of

307 specific antibodies in the sample allowing for quantification. Levels of IgM and IgG were 308 considered positive if greater than 50 relative fluorescence units. 
311 SARS-CoV-2 antibody neutralization titers were measured using a label-free surrogate

312 neutralization assay (LF-sVNT) previously described ${ }^{28}$. Briefly, the method measures the

313 binding ability of recombinant RBD (Sino Biological, Wayne, PA) coated onto sensing probes

314 (Gator Bio, Palo Alto, CA) to recombinant ACE2 (Sino Biological, Wayne, PA) after neutralizing

315 RBD with SARS-CoV-2 antibodies in serum. Measurements were done using a thin-film

316 interferometry (TFI) label-free immunoassay analyzer (Gator Bio, Palo Alto, CA). Each serum

317 sample was diluted in a series $(1: 25,1: 100,1: 250,1: 500,1: 1000,1: 2000)$ in running buffer

318 (PBS at $\mathrm{pH} 7.4$ with $0.02 \%$ Tween $20,0.2 \% \mathrm{BSA}$, and $0.05 \% \mathrm{NaN}_{3}$ ) for analysis. The first

319 testing cycle for each diluted sample measured the binding ability of RBD to ACE2 with

320 neutralization, and the second cycle provided the full binding ability of RBD without

321 neutralization. In each cycle, the recorded time course of signals, as known as the sensorgram,

322 was recorded. The readout measured the signal increase in RBD-ACE2 complex formation,

323 representing the quantity of RBD-ACE2 complex on the sensing probe. A neutralization rate

324 was calculated as the ratio of the readout in the first cycle to that in the second cycle, presented

325 as a percentage. To obtain the neutralizing antibody titer $\left(\mathrm{IC}_{50}\right)$ for each serum sample, the

326 neutralization rates were plotted against dilutions, and the points were fitted using a linear

327 interpolation model. The reciprocal of the dilution resulting in a $50 \%$ neutralization rate was

328 defined as the neutralizing antibody titer.

329

330 SARS-CoV-2 Spike protein Western blot. Maternal blood and cord blood were diluted in

331 RNAlater in 1:1.3 ratio, placenta was preserved in RNAlater. Protein lysates were obtained from 332 samples using RIPA buffer (150 mM NaCl, $25 \mathrm{mM}$ Tris- $\mathrm{HCl}(\mathrm{pH} 7.4), 1 \% \mathrm{NP}-40,0.5 \%$ sodium

333 deoxycholate, $0.1 \%$ sodium dodecyl sulfate) containing Halt ${ }^{\mathrm{TM}}$ protease inhibitor cocktail

334 (ThermoScientific).Cell Lysates were resolved by SDS/PAGE on a Bis-Tris methane 4-12\%

335 polyacrylamide gel and transferred to a nitrocellulose membrane, blocked with $5 \%$ skimmed 
milk diluted in PBS, an incubated overnight at $4^{\circ} \mathrm{C}$ with anti-SARS-CoV-2 Spike mouse mAb

337 (1A9, GeneTex) or anti-GAPDH rabbit polyclonal antibody (GTX100118, GeneTex) respectively

338 diluted 1:1,000 or 1:5,000 in blocking buffer. The membrane was washed in PBS buffer

339 containing Tween-20 (0.1\%) and then incubated for $1 \mathrm{~h}$ with horseradish peroxidase-conjugated

340 anti-mouse and anti-rabbit secondary antibody (Jackson ImmunoResearch) diluted respectively

$3411: 5,000$ and 1:10,000. The membrane was thoroughly washed, and proteins visualized using

342 Immobilon Forte Western HRP substrate (Millipore).

344 SARS-CoV-2 Spike mRNA PCR. Maternal blood and cord blood were diluted in RNAlater in

345 1:1.3 ratio, placenta was preserved in RNAlater. Tissues were kept at $-80^{\circ} \mathrm{C}$ until analyzed.

346 RNA was isolated from samples using the RNeasy Micro or Mini Kit (Qiagen) according to

347 manufacturer's protocol. RNA concentration was measured using nanodrop and all samples had

$348>30 \mathrm{ng} / \mathrm{ul}$ total RNA. 500ng RNA was transcribed into cDNA using qScript cDNA synthesis kit

349 (Quantabio). Primers were design to detect the vaccines mRNA (mRNA-1273 Moderna and

350 BNT162b2 Pfizer-BioNtech) as previously described ${ }^{36}$. Forward primer:

351 AACGCCACCAACGTGGTCATC. Reverse primer: GTTGTTGGCGCTGCTGTACAC. Primers

352 were shown to detect samples containing as low as $1.5 \mathrm{pg}$ of vaccine using vaccine standard

353 curve (Table S2). QuantaStudio 6 Flex (Applied Biosystems) instrument and SsoFast EvaGreen

354 supermix (Bio-Rad) were used for PCR reaction: 30 second $95^{\circ} \mathrm{C}$ followed by 40 cycles of 5

355 second $95^{\circ} \mathrm{C}$ and 20 seconds $60^{\circ} \mathrm{C}$. All samples were run in triplicate as $20 \mu \mathrm{L}$ reactions, and Ct

356 values corresponding to $<1.5 \mathrm{pg}$ of vaccine based on standard curve (Table S2) were

357 interpreted as a negative result. For vaccines cDNA standard curves, $10000 \mathrm{pg} / \mu \mathrm{L}$ vaccine

358 mRNA (as cDNA) sample was used for serial dilution in 1:3 ratio, up to $0.06 \mathrm{pg} / \mu \mathrm{L}$. Two $\mu \mathrm{L}$ of

359 these diluted samples were used in each well to create standard curves. 
$\underline{\text { Immunoprecipitation of phage-bound patient antibodies }}$

363 Maternal plasma at delivery and cord plasma were evaluated by PhIP-Seq/Virscan Coronavirus 364 phage display. Construction of the Coronavirus PhIP-Seq library and detailed methods for

365 immunoprecipitation, sequencing and bioinformatic processing of data are identical to what has 366 previously been described ${ }^{18}$. For the purposes of the analysis conducted in this study, analysis 367 was restricted to sero-reactivity against the SARS-CoV-2 Spike protein. As previously 368 described, a total of two rounds of amplification and selection were performed for all PhIP-Seq 369 analyses.

$371 \quad$ Next Generation Sequencing library prep

372 Amplicon sequencing library preps were performed using the Labcyte Echo 525 and an Integra

373 Via Flow 96 and were identical to what has previously been described ${ }^{18}$. All libraries were

374 pooled by equal volume, cleaned and size selected using Ampure XP beads at 1.0X per

375 manufacturer's protocol. Libraries were quantified by High Sensitivity DNA Qubit and quality-

376 checked by High Sensitivity DNA Bioanalyzer. Sequencing was then performed on a NovaSeq

377 S1 (300 cycle kit with 1.3 billion clusters) aiming for sequencing depths of at least 1 million 378 reads per sample.

Bioinformatic Analysis of PhIP-Seq Data

381 Sequencing reads were aligned to a reference database of the full viral peptide library using the 382 Bowtie2 aligner. For all VirScan libraries, the null distribution of each peptide's log10(rpK) was 383 modeled using a set of 95 pre-pandemic, healthy control sera. All counts were augmented by 1 384 to avoid zero counts in the healthy control sera samples. Multiple distribution fits were examined 385 for these data, with the Normal distribution showing the best fit. These null distributions were 386 used to calculate $\mathrm{p}$ values for the observed log10(rpK) of each peptide within a given sample. 387 The calculated $p$ values were corrected for multiple hypothesis using the Benjamini-Hochberg 
method. Any peptide with a corrected $p$ value of $<0.001$ was considered significantly enriched over the healthy background. To identify regions targeted by host antibodies, all library peptides were aligned to the SARS-CoV-2 reference genome. Using the aligned position of the

391 significantly enriched peptides which aligned full-length against the reference, we determined 392 the proportion of individuals (mothers and infants) that were reactive at each residue of the 393 Spike protein. All plots were generated using the R ggplot2 package.

\section{Statistical analysis:}

396 Statistical analyses were performed using PRISM v9.2 (GraphPad), STATA 16 (StataCorp), and $397 \mathrm{R}$ version 3.6.3 and $\mathrm{R}$ Studio version 1.1.447. Descriptive statistics include mean, standard 398 deviations, and ranges for continuous variables. The Wilcoxon rank-sum test was used for two399 group comparisons of continuous variables including maternal pre- and post-vaccine antibody 400 responses. Associations between continuous variables were assessed using Spearman's rank 401 correlation $\left(R_{s}\right)$ including comparisons between maternal, cord and infant follow-up antibody lgG 402 and neutralizing titer responses, and timing of vaccination. Two-sided $p$ values were calculated 403 for all test statistics, and $p<0.05$ was considered significant. PhIP-Seq/VirScan bioinformatics as 404 detailed above.

Data Availability

407 The data set generated during and/or analyzed during the current study are available from the 408 corresponding author on reasonable request.

\section{Acknowledgements:}

411 M.P. was supported by the National Institutes of Health (NIAID K23AI127886), the Marino

412 Family Foundation, and UCSF REAC award. Y.G. was supported by the Weizmann Institute of 413 Science -National Postdoctoral Award Program for Advancing Women in Science, and of the 
414 International Society for Research in Human Milk and Lactation (ISRHML) Trainee Bridge Fund.

415 Y.M. and W.C.G. were supported by The Roddenberry Foundation. S.L.G. was supported by

416 the National Institutes of Health (NIAID K08AI141728), and the Bill and Melinda Gates

417 Foundation (INV-017035), and a generous gift from the Kryzewski Family.

418

419 We thank all the mothers and infants that participated in this study. We thank Kenneth Scott, 420 BS, RPh, (UCSF Health Pharmacy, University of California, San Francisco) and Hannah J.

421 Jang, PhD, RN, PHN, CNL (UCSF School of Nursing, University of California, San Francisco),

422 for voluntarily providing unused vaccine for this study, and to Dr. Margaret Feeney (University of 423 California, San Francisco) and Dr. Nadav Ahituv (University of California, San Francisco) for 424 support of these experiments.

425

426 Author contributions:

427 M.P. Helped conceive and design the project, oversaw recruitment, designed, and performed

428 sample collection, oversaw experiment design, oversaw data analysis, provided funding, and

429 drafted the manuscript.

430 Y.G. Recruited and consented enrollees, oversaw sample collection, designed, performed, and 431 analyzed mRNA PCR experiments, performed data analysis.

432 A.G.C. Recruited and consented enrollees, oversaw sample collection, performed chart review, 433 and helped draft the manuscript.

434 Y.M. Performed and helped design Western blot.

435 L.L. Performed and analyzed mRNA PCR experiments, performed sample collection.

436 B.A. Performed phage immunoprecipitation assays.

437 H.C. and U.J. performed and helped design critical experiments, and data collection.

438 C.Y.L., V.J.L., M.C., L.W., S.B. Performed and coordinated sample collection, and data collection. 
439 V.J.F. Helped conceive and coordinate the project.

440 A.P.M. Provide funding.

441 W.C.G. Helped design western blot and oversaw data analysis.

442 A.H.B.W Designed and oversaw all serology experiments.

443 K.L.L. Designed and oversaw all neutralizing antibody experiments.

444 J.R. Designed, analyzed, and oversaw phage immunoprecipitation sequencing assays.

445 S.L.G. conceived and designed the project, oversaw recruitment, oversaw experiment design, 446 oversaw data analysis, provided funding, and helped draft the manuscript.

447 M.P., Y.G., Y.U., L.L., A.H.B.W, W.C.G, K.L.L., and S.L.G verified the underlying data.

448 All authors reviewed and approved the manuscript.

449

450

451

452

453

454

455

456

457

458

459

460

461 
A.

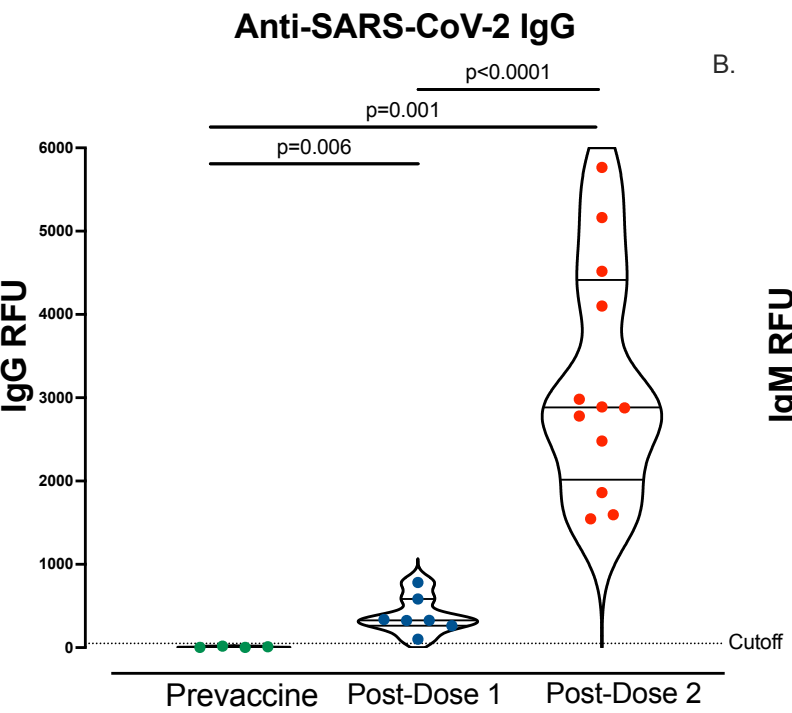

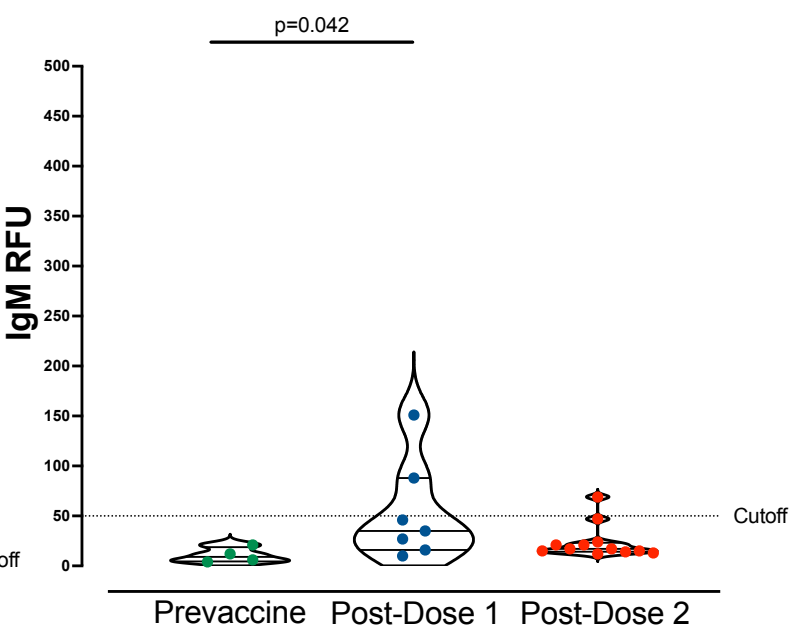

463 Figure 1. Anti-SARS-CoV-2 IgG and IgM antibody responses following vaccination

464 A. Maternal plasma anti-SARS-CoV-2 IgG antibody relative fluorescence units (RFU) levels

465 prior to vaccination ( $n=4), 3-4$ weeks post-dose $1(n=7)$, and 4-8 weeks post-dose $2(n=12)$. B.

466 Maternal plasma anti-SARS-CoV-2 IgM (RFU) levels prior to vaccination ( $n=4)$, 3-4 weeks post-

467 dose $1(n=7)$, and 4-8 weeks post-dose $2(n=12)$. Wilcoxon rank-sum testing. Data represent

468 median \pm quartiles, two-sided $p$ values were calculated for all test statistics.

469

470

471

472

473

474

475

476

477 

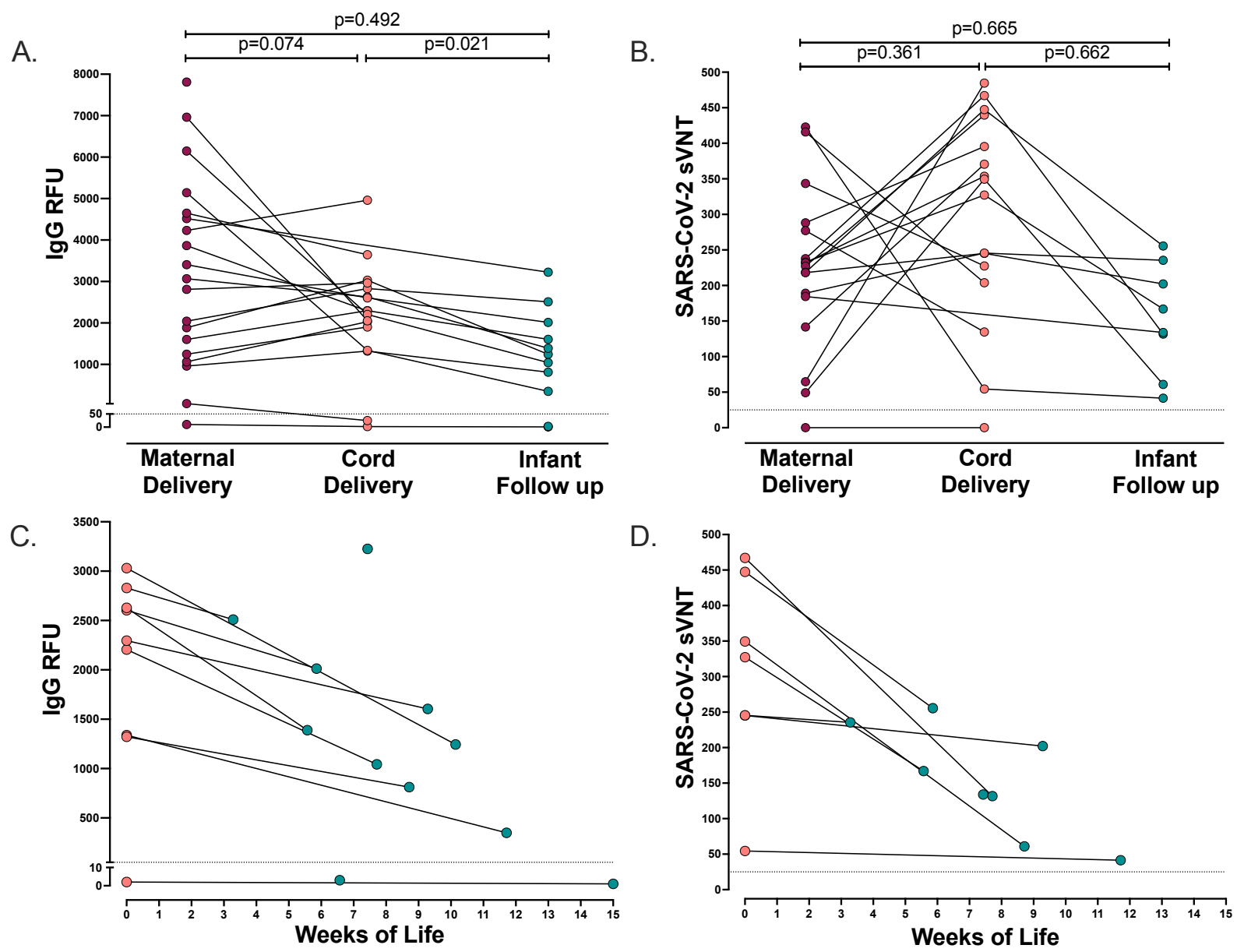

E.

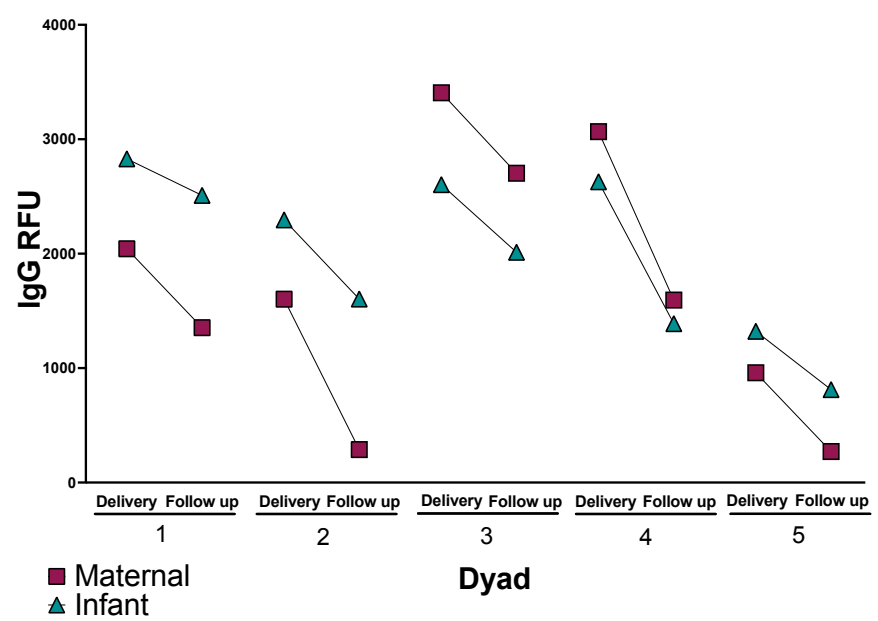

Figure 2. Paired maternal, cord, and infant IgG and neutralization antibodies

481 A. Paired maternal plasma at delivery $(n=19)$, cord plasma $(n=17)$, and infant follow-up $(n=10)$

482 by anti-SARS-CoV-2 IgG antibody relative fluorescence units (RFU), (Spearman's rank

483 correlation, dotted line indicates positive cutoff value of 50 RFU). B. Paired maternal plasma at 
484 delivery $(n=17)$, cord plasma $(n=16)$, and infant follow-up $(n=8)$ by SARS-CoV-2 label-free 485 surrogate neutralization assay (sVNT), (Spearman's rank correlation, dotted line indicates 486 positive cutoff value of 25). C. Paired cord plasma $(n=9)$ and infant follow-up plasma $(n=11)$ 487 anti-SARS-CoV-2 IgG by weeks of life. D. Paired cord plasma $(n=7)$ and infant follow-up plasma $488(n=8)$ label-free surrogate neutralization assay (sVNT) by weeks of life. E. Paired maternal 489 plasma at delivery $(n=5)$, cord plasma $(n=5)$, and paired maternal follow-up $(n=5)$ and infant 490 follow-up plasma $(n=5)$ anti-SARS-CoV-2 IgG. Two-sided $p$ values were calculated for all test 491 statistics.

492

493

494

495

496

497

498

499

500

501

502 
A. Maternal Plasma at Delivery

B.



C. Infant Follow-up Plasma

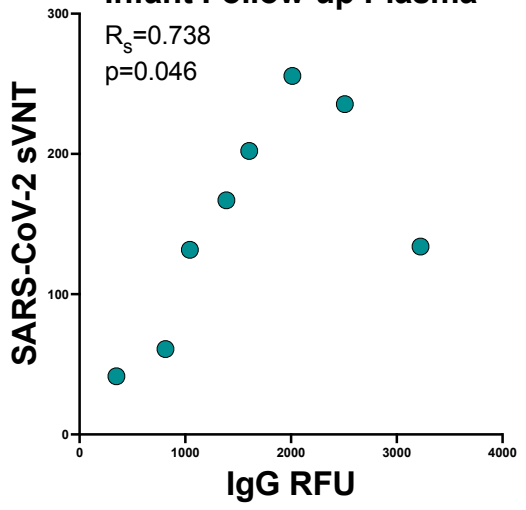

504 Figure 3. Neutralization to IgG antibody correlation

505 A. Maternal plasma at delivery $(n=17)$ B. Cord plasma $(n=16)$ C. Infant follow-up plasma $(n=8)$

506 SARS-CoV-2 label-free surrogate neutralization assay (sVNT) by anti-SARS-CoV-2 lgG

507 correlation (Spearman's rank correlation). Two-sided $p$ values were calculated for all test

508 statistics.

509

510

511

512

513

514

515

516

517

518

519

520

521 
Maternal Delivery

A.

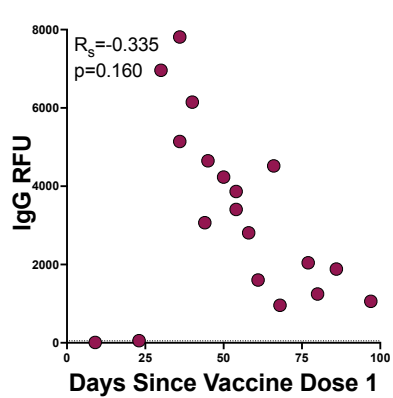

B.

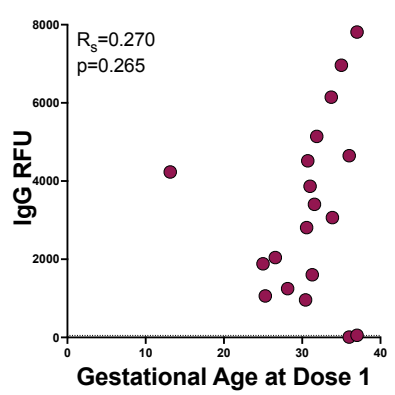

C.

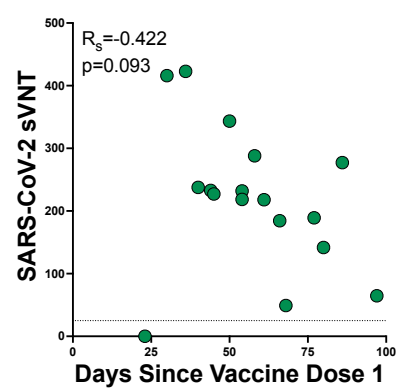

D.

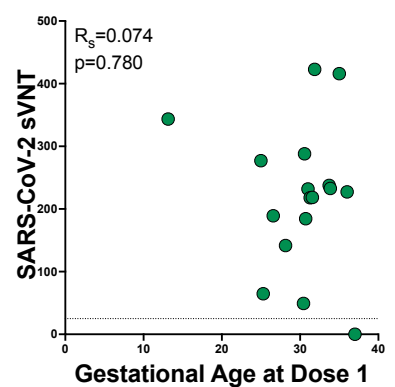

E.

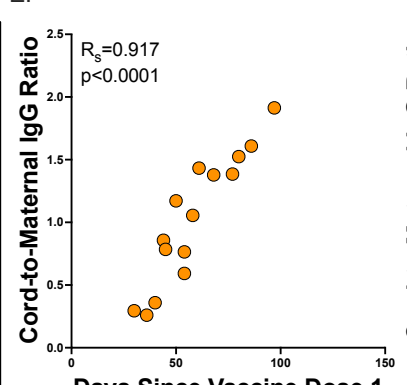

Days Since Vaccine Dose 1

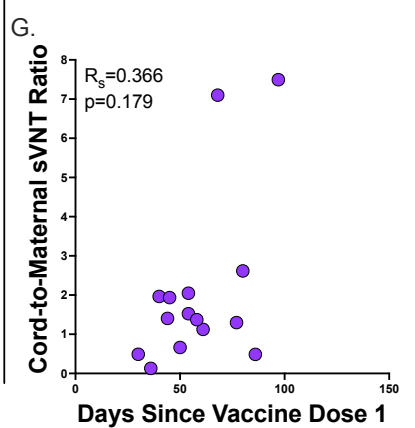

Cord-to-Maternal Ratio
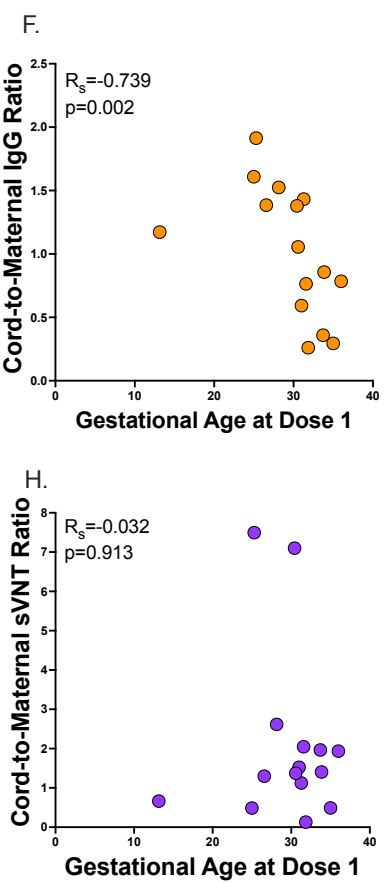

Figure 4. Maternal delivery and Cord-to-maternal antibody transfer ratios timing

524 A. Maternal delivery anti-SARS-CoV-2 IgG antibody transfer ratio by days since vaccine dose 1

525 ( $n=19$, dashed line indicates positive cutoff >50 RFU) B. Maternal delivery anti-SARS-CoV-2

526 IgG antibody transfer ratio by gestational age at vaccine dose $1(n=19$, dashed line indicates

527 positive cutoff $>50$ RFU) C. Maternal delivery SARS-CoV-2 label-free surrogate neutralization

528 assay (sVNT) antibody transfer ratio by days since vaccine dose $1(n=17$, dashed line indicates

529 positive cutoff $>25$ ). D. Maternal delivery SARS-CoV-2 label-free surrogate neutralization assay

530 (sVNT) antibody transfer ratio by gestational age at vaccine dose $1(n=17$, dashed line indicates

531 positive cutoff >25) E. Cord-to-maternal anti-SARS-CoV-2 IgG antibody transfer ratio by days

532 since vaccine dose $1(n=15)$ F. Cord-to-maternal anti-SARS-CoV-2 IgG antibody transfer ratio

533 by gestational age at vaccine dose $1(n=15)$ G. Cord-to-maternal SARS-CoV-2 label-free

534 surrogate neutralization assay (sVNT) antibody transfer ratio by days since vaccine dose 1

535 (n=15). H. Cord-to-maternal SARS-CoV-2 label-free surrogate neutralization assay (sVNT) 
536 antibody transfer ratio by gestational age at vaccine dose $1(n=15)$. Two-sided $p$ values were

537 calculated for all test statistics.

538

539

540

541

542

543

544

545

546

547

548

549

550

551

552

553

554

555

556

557

558

559

560

561 
A. Dyads $\quad$ Vaccine Days

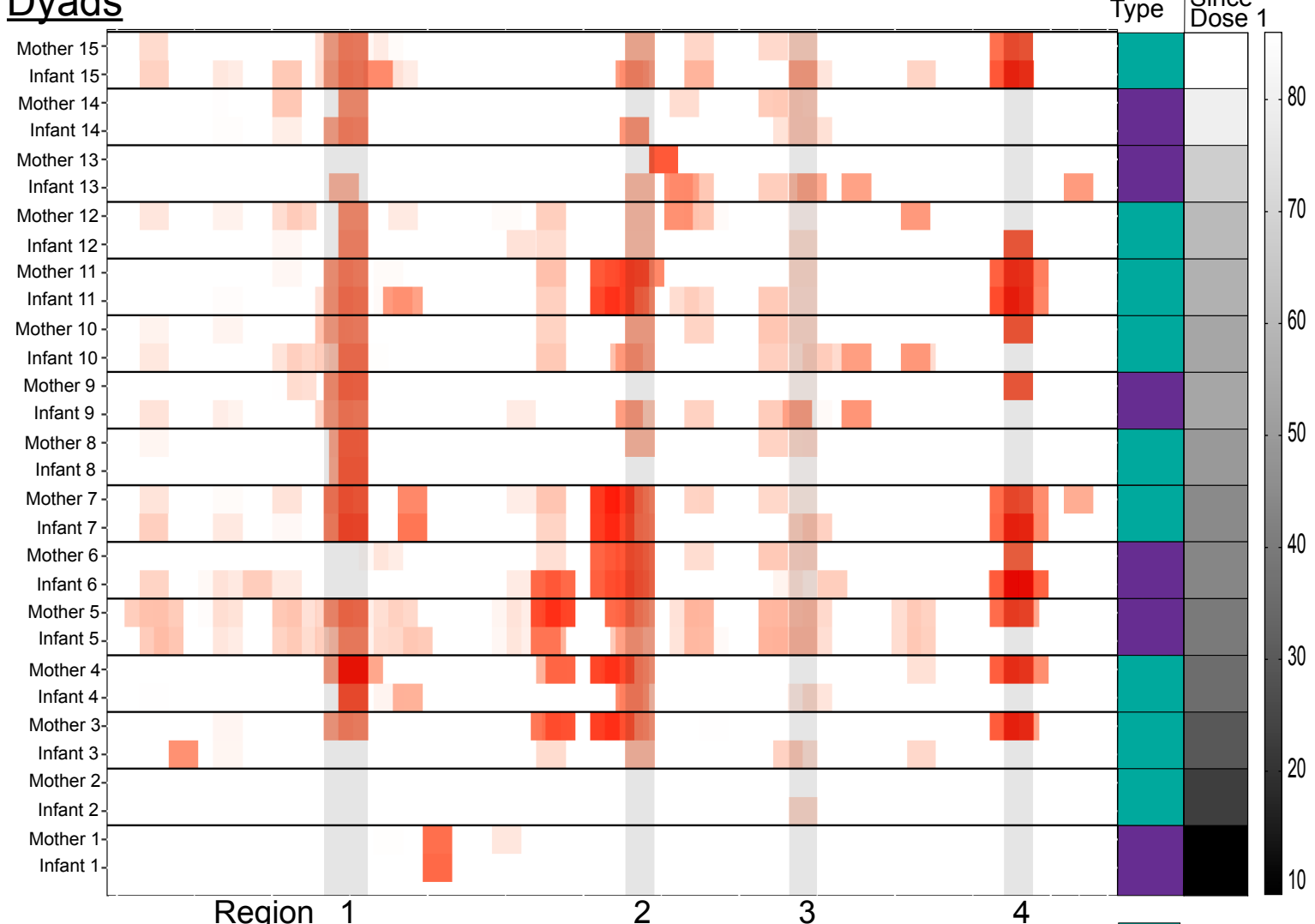

B.

$\log 10\left(\right.$ rp100k) $\begin{array}{lllll}0 & 1 & 2 & 3 & 4\end{array}$

mRNA-1273

BNT162b2
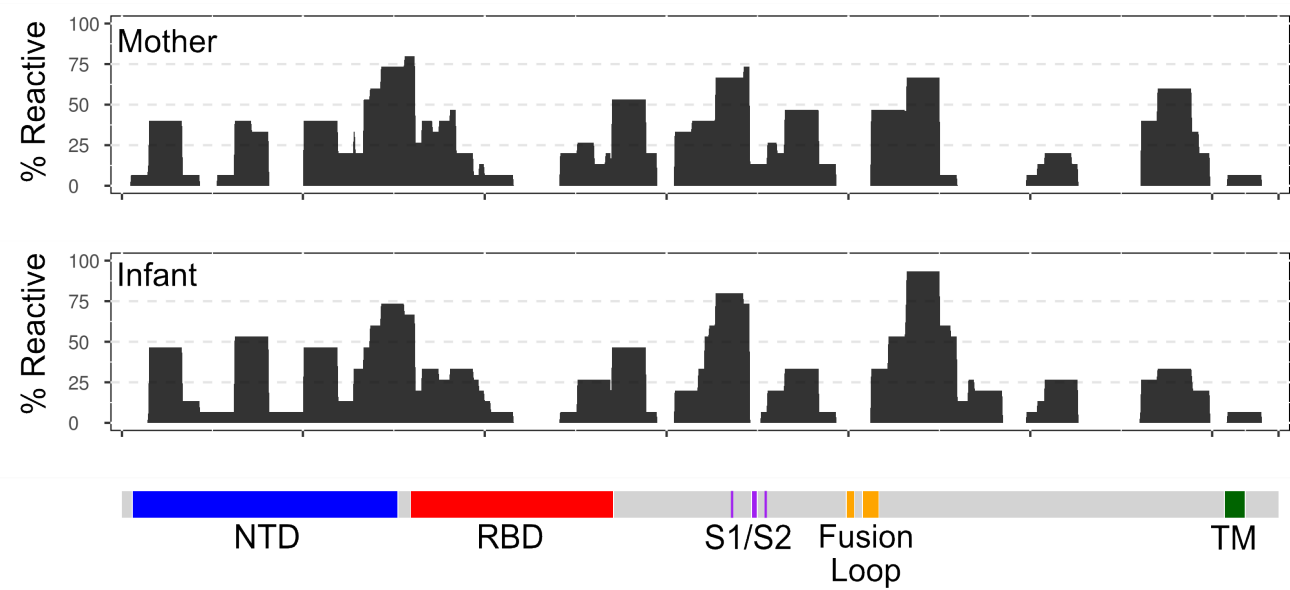

562 Figure 5. PhIP-seq/VirScan paired maternal and cord SARS-CoV-2 Spike protein epitope

563 binding

564 A. Heatmap displaying results of significant enriched $(p<0.001)$ linear SARS-CoV-2 Spike

565 protein epitope binding from 15 paired mother-infant dyads in maternal plasma at delivery and

566 cord plasma by vaccine type and time since vaccine dose 1. Areas of high cumulative epitope 
567 binding designated by regions 1-4. B. Cumulative fold enrichment of mothers and infants linear 568 SARS-CoV-2 Spike protein epitope binding.

569

570

571

572

573

574

575

576

577

578

579

580

581

582

583

584

585

586

587

588

589

590

591

592 
$\underline{\text { References: }}$

5941 Ellington, S. et al. Characteristics of Women of Reproductive Age with Laboratory-

595 Confirmed SARS-CoV-2 Infection by Pregnancy Status - United States, January 22-June 7,

596 2020. MMWR Morb Mortal Wkly Rep 69, 769-775, doi:10.15585/mmwr.mm6925a1

597 (2020).

5982 Ahlberg, M. et al. Association of SARS-CoV-2 Test Status and Pregnancy Outcomes.

$599 \quad$ Jama, doi:10.1001/jama.2020.19124 (2020).

6003 Knight, M. et al. Characteristics and outcomes of pregnant women admitted to hospital

601 with confirmed SARS-CoV-2 infection in UK: national population based cohort study. Bmj

$602 \quad 369$, m2107, doi:10.1136/bmj.m2107 (2020).

6034 Martinez-Portilla, R. J. et al. Pregnant women with SARS-CoV-2 infection are at higher

604 risk of death and pneumonia: propensity score matched analysis of a nationwide

605 prospective cohort (COV19Mx). Ultrasound Obstet Gynecol 57, 224-231,

606 doi:10.1002/uog.23575 (2021).

6075 CDC. COVID-19 vaccination among pregnant people aged 18-49 years overall, by

608 race/ethnicity, and date reported to CDC - Vaccine Safety Datalink, * United States,

$609 \quad<$ https://covid.cdc.gov/covid-data-tracker/\#vaccinations-pregnant-women> (2021).

6106 Gynecologists, A. C. o. O. a. Statement of Strong Medical Consensus for Vaccination of

$611 \quad$ Pregnant Individuals Against COVID-19, <https://www.acog.org/news/news-

612 releases/2021/08/statement-of-strong-medical-consensus-for-vaccination-of-pregnant-

$613 \quad$ individuals-against-covid-19> (2021). 
6147 Maykin, M. M., Heuser, C., Feltovich, H. \& with the Society for Maternal-Fetal Medicine

615 Health Policy Advocacy, C. Pregnant people deserve the protection offered by SARS-

616 CoV-2 vaccines. Vaccine 39, 171-172, doi:10.1016/j.vaccine.2020.12.007 (2021).

6178 Jackson, L. A. et al. An mRNA Vaccine against SARS-CoV-2 - Preliminary Report. N Engl J

$618 \quad$ Med 383, 1920-1931, doi:10.1056/NEJMoa2022483 (2020).

6199 Polack, F. P. et al. Safety and Efficacy of the BNT162b2 mRNA Covid-19 Vaccine. N Engl J

$620 \quad$ Med 383, 2603-2615, doi:10.1056/NEJMoa2034577 (2020).

62110 Atyeo, C. et al. Compromised SARS-CoV-2-specific placental antibody transfer. Cell, 622 doi:10.1016/j.cell.2020.12.027 (2020).

62311 Edlow, A. G. et al. Assessment of Maternal and Neonatal SARS-CoV-2 Viral Load,

624 Transplacental Antibody Transfer, and Placental Pathology in Pregnancies During the 625 COVID-19 Pandemic. JAMA Netw Open 3, e2030455,

626 doi:10.1001/jamanetworkopen.2020.30455 (2020).

62712 Flannery, D. D. et al. Assessment of Maternal and Neonatal Cord Blood SARS-CoV-2 628 Antibodies and Placental Transfer Ratios. JAMA Pediatr, 629 doi:10.1001/jamapediatrics.2021.0038 (2021).

63013 Atyeo, C. et al. Compromised SARS-CoV-2-specific placental antibody transfer. Cell 184, 631 628-642.e610, doi:10.1016/j.cell.2020.12.027 (2021).

63214 Gray, K. J. et al. Coronavirus disease 2019 vaccine response in pregnant and lactating 633 women: a cohort study. Am J Obstet Gynecol 225, 303.e301-303.e317, 634 doi:10.1016/j.ajog.2021.03.023 (2021).

63515 Nir, O. et al. Maternal-neonatal transfer of SARS-CoV-2 immunoglobulin G antibodies 636 among parturient women treated with BNT162b2 messenger RNA vaccine during 

(2021).

63916 Song, D. et al. Passive and active immunity in infants born to mothers with SARS-CoV-2 640 infection during pregnancy: prospective cohort study. BMJ Open 11, e053036, doi:10.1136/bmjopen-2021-053036 (2021).

64217 Atyeo, C. et al. COVID-19 mRNA vaccines drive differential antibody Fc-functional 643 644 doi:10.1126/scitranslmed.abi8631 (2021).

64518 Zamecnik, C. R. et al. ReScan, a Multiplex Diagnostic Pipeline, Pans Human Sera for 646 SARS-CoV-2 Antigens. Cell Rep Med 1, 100123, doi:10.1016/j.xcrm.2020.100123 (2020).

64719 Shrock, E. et al. Viral epitope profiling of COVID-19 patients reveals cross-reactivity and 648 correlates of severity. Science 370, doi:10.1126/science.abd4250 (2020).

$64920 \mathrm{Li}$, Y. et al. Linear epitopes of SARS-CoV-2 spike protein elicit neutralizing antibodies in 650 COVID-19 patients. Cell Mol Immunol 17, 1095-1097, doi:10.1038/s41423-020-00523-5

651 (2020).

65221 Fenizia, C. et al. Analysis of SARS-CoV-2 vertical transmission during pregnancy. Nat Commun 11, 5128, doi:10.1038/s41467-020-18933-4 (2020).

65422 Zeng, H. et al. Antibodies in Infants Born to Mothers With COVID-19 Pneumonia. JAMA 655 323, 1848-1849, doi:10.1001/jama.2020.4861 (2020).

65623 Zeng, L. et al. Neonatal Early-Onset Infection With SARS-CoV-2 in 33 Neonates Born to 657 Mothers With COVID-19 in Wuhan, China. JAMA Pediatr 174, 722-725, 658 doi:10.1001/jamapediatrics.2020.0878 (2020). 
65924 Healy, C. M. et al. Kinetics of maternal pertussis-specific antibodies in infants of mothers vaccinated with tetanus, diphtheria and acellular pertussis (Tdap) during pregnancy.

661 Vaccine 38, 5955-5961, doi:10.1016/j.vaccine.2020.06.050 (2020).

66225 Sissoko, M. S. et al. Safety and efficacy of PfSPZ Vaccine against Plasmodium falciparum 663 via direct venous inoculation in healthy malaria-exposed adults in Mali: a randomised, 664 double-blind phase 1 trial. The Lancet Infectious Diseases 17, 498-509, 665 doi:10.1016/s1473-3099(17)30104-4 (2017).

66626 Leuridan, E., Hens, N., Hutse, V., Aerts, M. \& Van Damme, P. Kinetics of maternal 667 antibodies against rubella and varicella in infants. Vaccine 29, 2222-2226,

668 doi:10.1016/j.vaccine.2010.06.004 (2011).

669

27 Pyzik, M. et al. The Neonatal Fc Receptor (FcRn): A Misnomer? Front Immunol 10, 1540, 670 doi:10.3389/fimmu.2019.01540 (2019).

67128 Luo, Y. R., Yun, C., Chakraborty, I., Wu, A. H. B. \& Lynch, K. L. A SARS-CoV-2 Label-Free 672 Surrogate Virus Neutralization Test and a Longitudinal Study of Antibody Characteristics

673 in COVID-19 Patients. J Clin Microbiol 59, e0019321, doi:10.1128/jcm.00193-21 (2021).

67429 Firan, M. et al. The MHC class I-related receptor, FcRn, plays an essential role in the 675 maternofetal transfer of gamma-globulin in humans. Int Immunol 13, 993-1002, 676 doi:10.1093/intimm/13.8.993 (2001).

67730 Williams, P. J. et al. Short communication: selective placental transport of maternal IgG 678 to the fetus. Placenta 16, 749-756, doi:10.1016/0143-4004(95)90018-7 (1995).

67931 Kibe, T. et al. Glycosylation and Placental Transport of Immunoglobulin G. Journal of 680 Clinical Biochemistry and Nutrition 21, 57-63 (1996). 
68132 Borghi, S. et al. FcRn, but not FcpRs, drives maternal-fetal transplacental transport of 682 human IgG antibodies. Proc Natl Acad Sci U S A 117, 12943-12951, 683 doi:10.1073/pnas.2004325117 (2020).

68433 Palmeira, P., Quinello, C., Silveira-Lessa, A. L., Zago, C. A. \& Carneiro-Sampaio, M. IgG 685 placental transfer in healthy and pathological pregnancies. Clin Dev Immunol 2012, 686 985646, doi:10.1155/2012/985646 (2012).

68734 Clements, T. et al. Update on Transplacental Transfer of IgG Subclasses: Impact of 688 Maternal and Fetal Factors. Front Immunol 11, 1920, doi:10.3389/fimmu.2020.01920 689 (2020).

69035 Chi, X. et al. A neutralizing human antibody binds to the N-terminal domain of the Spike 691 protein of SARS-CoV-2. Science 369, 650-655, doi:10.1126/science.abc6952 (2020).

69236 Golan, Y. et al. Evaluation of Messenger RNA From COVID-19 BTN162b2 and mRNA-1273

693 Vaccines in Human Milk. JAMA Pediatr 175, 1069-1071,

694 doi:10.1001/jamapediatrics.2021.1929 (2021).

695 


\section{Supplementary Files}

This is a list of supplementary files associated with this preprint. Click to download.

- SupplementalAppendixEvaluationoftransplacentalofmRNAvaccineproductsandfunctionalantibodiesduringpregnancyandearlyinfancy.pdf 